\title{
UNIVERSAL DEFORMATION RINGS AND DIHEDRAL 2-GROUPS
}

\author{
FRAUKE M. BLEHER
}

\begin{abstract}
Let $k$ be an algebraically closed field of characteristic 2, and let $W$ be the ring of infinite Witt vectors over $k$. Suppose $D$ is a dihedral 2-group. We prove that the universal deformation ring $R(D, V)$ of an endo-trivial $k D$-module $V$ is always isomorphic to $W[\mathbb{Z} / 2 \times \mathbb{Z} / 2]$. As a consequence we obtain a similar result for modules $V$ with stable endomorphism ring $k$ belonging to an arbitrary nilpotent block with defect group $D$. This confirms for such $V$ conjectures on the ring structure of the universal deformation ring of $V$ which had previously been shown for $V$ belonging to cyclic blocks or to blocks with Klein four defect groups.
\end{abstract}

\section{INTRODUCTION}

Let $k$ be an algebraically closed field of positive characteristic $p$, let $W=W(k)$ be the ring of infinite Witt vectors over $k$, and let $G$ be a finite group. There are various classical results in the literature concerning the lifting of finitely generated $k G$-modules over complete local commutative Noetherian rings with residue field $k$, such as Green's liftability theorem. To understand and generalize these results, it is useful to reformulate them in terms of deformation rings. For example, Alperin has proved in [2] that in case $G$ is a $p$-group, every endo-trivial $k G$-module $V$ can be lifted to an endo-trivial $W G$-module. This can be reformulated as saying that for every such $V$, there is a surjection from the universal deformation ring $R(G, V)$ to $W$. A natural question is then to determine $R(G, V)$ itself. In this paper, we determine the universal deformation rings $R(D, V)$ for all endo-trivial $k D$-modules $V$ when $k$ has characteristic $p=2$ and $D$ is a dihedral 2-group.

For arbitrary $p$ and $G$, a finitely generated $k G$-module $V$ is called endo-trivial if the $k G$-module $\operatorname{Hom}_{k}(V, V) \cong V^{*} \otimes_{k} V$ is isomorphic to a direct sum of the trivial simple $k G$-module $k$ and a projective $k G$-module. Endo-trivial modules play an important role in the modular representation theory of finite groups, in particular in the context of derived equivalences and stable equivalences of block algebras, and also as building blocks for the more general endo-permutation modules, which for many groups are the sources of the simple modules (see e.g. [17, 27]). In [15, 14], Carlson and Thévenaz classified all endo-trivial $k G$-modules when $G$ is a $p$-group. Since by [12], the endo-trivial $k G$-modules $V$ of a $p$-group $G$ are precisely the modules whose

1991 Mathematics Subject Classification. Primary 20C20; Secondary 20C15, 16 G10.

Key words and phrases. Universal deformation rings, dihedral groups, endo-trivial modules, nilpotent blocks.

The author was supported in part by NSA Grant H98230-06-1-0021 and NSF Grant DMS0651332 . 
stable endomorphism ring is one-dimensional over $k$, it follows by [6, Prop. 2.1] that $V$ has a well-defined universal deformation $\operatorname{ring} R(G, V)$.

The topological ring $R(G, V)$ is universal with respect to deformations of $V$ over complete local commutative Noetherian rings $R$ with residue field $k$. A deformation of $V$ over such a ring $R$ is given by the isomorphism class of a finitely generated $R G$ module $M$ which is free over $R$, together with a $k G$-module isomorphism $k \otimes_{R} M \rightarrow V$ (see 92$)$. Note that all these rings $R$, including $R(G, V)$, have a natural structure as $W$-algebras.

In number theory, the main motivation for studying universal deformation rings for finite groups is to provide evidence for and counter-examples to various possible conjectures concerning ring theoretic properties of universal deformation rings for profinite Galois groups. The idea is that universal deformation rings for finite groups can be more easily described using deep results from modular representation theory due to Brauer, Erdmann [19], Linckelmann [21, 22], Butler-Ringel [10] and others. Moreover, the results in 18 show that if $\Gamma$ is a profinite group and $V$ is a finite dimensional $k$-vector space with a continuous $\Gamma$-action which has a universal deformation ring, then $R(\Gamma, V)$ is the inverse limit of the universal deformation rings $R(G, V)$ when $G$ runs over all finite discrete quotients of $\Gamma$ through which the $\Gamma$ action on $V$ factors. Thus to answer questions about the ring structure of $R(\Gamma, V)$, it is natural to first consider the case when $\Gamma=G$ is finite. Later in the introduction we will discuss some number theoretic problems which originate from considering how our results for finite groups arise from arithmetic.

Suppose now that $G$ is an arbitrary finite group and $V$ is a $k G$-module such that the stable endomorphism ring $\underline{\operatorname{End}}_{k G}(V)$ is one-dimensional over $k$, i.e. $V$ has a welldefined universal deformation ring $R(G, V)$. The results in [6] led to the following question relating the universal deformation $\operatorname{rings} R(G, V)$ to the local structure of $G$ given by defect groups of blocks of $k G$.

Question 1.1. Let $B$ be a block of $k G$ with defect group $D$, and suppose $V$ is a finitely generated $k G$-module with stable endomorphism ring $k$ such that the unique (up to isomorphism) non-projective indecomposable summand of $V$ belongs to $B$. Is the universal deformation ring $R(G, V)$ of $V$ isomorphic to a subquotient ring of the group ring $W D$ ?

The results in [6, 4, 5] show that this question has a positive answer in case $B$ is a block with cyclic defect groups, i.e. a block of finite representation type, or a tame block with Klein four defect groups, or a tame block with dihedral defect groups which is Morita equivalent to the principal 2-modular block of a finite simple group. For the latter type of blocks, there are precisely three isomorphism classes of simple modules.

In [7, 8], it was shown that if $p=2, G$ is the symmetric group $S_{4}$ and $E$ is a 2-dimensional simple $k S_{4}$-module then $R(G, E) \cong W[t] /\left(t^{2}, 2 t\right)$, giving an example of a universal deformation ring which is not a complete intersection, thus answering a question of M. Flach [16]. A new proof of this result has been given in [11] using only elementary obstruction calculus. In [7, 8], it was additionally shown that this example arises from arithmetic in the following way. There are infinitely many real quadratic 
fields $L$ such that the Galois group $G_{L, \emptyset}$ of the maximal totally unramified extension of $L$ surjects onto $S_{4}$ and $R\left(G_{L, \emptyset}, E\right) \cong R\left(S_{4}, E\right) \cong W[t] /\left(t^{2}, 2 t\right)$ is not a complete intersection, where $E$ is viewed as a module of $G_{L, \emptyset}$ via inflation. The universal deformation rings in [6, 4] are all complete intersections, whereas the results in [5] provide an infinite series of $G$ and $V$ for which $R(G, V)$ is not a complete intersection.

In this paper, we consider the endo-trivial modules for the group ring $k D$ when $k$ has characteristic 2 and $D$ is a dihedral 2-group of order at least 8 . Note that $k D$ is its own block and the trivial simple module $k$ is the unique simple $k D$-module up to isomorphism. Our main result is as follows, where $\Omega$ denotes the syzygy, or Heller, operator (see for example [1, §20]).

Theorem 1.2. Let $k$ be an algebraically closed field of characteristic 2 , let $d \geq 3$, and let $D$ be a dihedral group of order $2^{d}$. Suppose $V$ is a finitely generated endo-trivial $k D$-module.

i. If $V$ is indecomposable and $\mathfrak{C}$ is the component of the stable Auslander-Reiten quiver of $k D$ containing $V$, then $\mathfrak{C}$ contains either $k$ or $\Omega(k)$, and all modules belonging to $\mathfrak{C}$ are endo-trivial.

ii. The universal deformation ring $R(D, V)$ is isomorphic to $W[\mathbb{Z} / 2 \times \mathbb{Z} / 2]$. Moreover, every universal lift $U$ of $V$ over $R=R(D, V)$ is endo-trivial in the sense that the $R D$-module $U^{*} \otimes_{R} U$ is isomorphic to the direct sum of the trivial $R D$-module $R$ and a free $R D$-module.

In particular, $R(D, V)$ is always a complete intersection and isomorphic to a quotient ring of the group ring $W D$.

It is a natural question to ask whether Theorem 1.2 can be used to construct deformation rings arising from arithmetic. More precisely, let $L$ be a number field, let $S$ be a finite set of places of $L$, and let $L_{S}$ be the maximal algebraic extension of $L$ unramified outside $S$. Denote by $G_{L, S}$ the Galois group of $L_{S}$ over $L$. Suppose $k$ has characteristic $p, G$ is a finite group and $V$ is a finitely generated $k G$-module with stable endomorphism ring $k$. As in [8], one can ask whether there are $L$ and $S$ such that there is a surjection $\psi: G_{L, S} \rightarrow G$ which induces an isomorphism of deformation rings $R\left(G_{L, S}, V\right) \rightarrow R(G, V)$ when $V$ is viewed as a representation for $G_{L, S}$ via $\psi$. It was shown in [8] that a sufficient condition for $R\left(G_{L, S}, V\right) \rightarrow R(G, V)$ to be an isomorphism for all such $V$ is that $\operatorname{Ker}(\psi)$ has no non-trivial pro- $p$ quotient. If this condition is satisfied, we say the group $G$ caps $L$ for $p$ at $S$.

As mentioned earlier, this arithmetic problem was considered in [8] for the symmetric group $S_{4}$ in case $p=2$. In fact, it was shown that there are infinitely many real quadratic fields $L$ such that $S_{4}$ caps $L$ for $p=2$ at $S=\emptyset$. Since the Sylow 2-subgroups of $S_{4}$ are isomorphic to a dihedral group $D_{8}$ of order 8 , this can be used to show that $D_{8}$ caps infinitely many sextic fields $L^{\prime}$ for $p=2$ at $S=\emptyset$. In particular, $R\left(G_{L^{\prime}, \emptyset}, V\right) \cong R\left(D_{8}, V\right) \cong W[\mathbb{Z} / 2 \times \mathbb{Z} / 2]$ for all endo-trivial $k D_{8}$-modules $V$. Since the fields $L^{\prime}$ have degree 6 over $\mathbb{Q}$, this raises the question of whether one can replace $L^{\prime}$ by smaller degree extensions. Another question is if one can find similar results for dihedral groups $D$ of arbitrary 2-power order. As in the proof of [8, Thm. $3.7(\mathrm{i})$ ], one can show that $D$ does not cap $\mathbb{Q}$ for $p=2$ at any finite set $S$ of rational 
primes. Hence the best possible results one can expect to be valid for all endo-trivial $k D$-modules should involve extensions $L$ of $\mathbb{Q}$ of degree at least 2 .

We now discuss the proof of Theorem 1.2. As stated earlier, the endo-trivial $k D$-modules are precisely the $k D$-modules whose stable endomorphism ring is onedimensional over $k$. The results of [13, §5] show that the group $T(D)$ of equivalence classes of endo-trivial $k D$-modules is generated by the classes of the relative syzygies of the trivial simple $k D$-module $k$. To prove part (i) of Theorem 1.2 we relate this description for indecomposable endo-trivial $k D$-modules to their location in the stable Auslander-Reiten quiver of $k D$. For part (ii) of Theorem 1.2, suppose $D=\langle\sigma, \tau\rangle$ where $\sigma$ and $\tau$ are two elements of order 2 and $\sigma \tau$ has order $2^{d-1}$, and let $V$ be an indecomposable endo-trivial $k D$-module. We prove that there exists a continuous local $W$-algebra homomorphism $\alpha: W[\mathbb{Z} / 2 \times \mathbb{Z} / 2] \rightarrow R(D, V)$ by considering restrictions of $V$ to $\langle\sigma\rangle$ and $\langle\tau\rangle$. We then analyze the $k D$-module structures of all lifts of $V$ over the dual numbers $k[\epsilon] /\left(\epsilon^{2}\right)$ to show that $\alpha$ is in fact surjective. Using the ordinary irreducible representations of $D$, we prove that there are four distinct continuous $W$-algebra homomorphisms $R(D, V) \rightarrow W$ and show that this implies that $\alpha$ is an isomorphism.

In [9, 25], Broué and Puig introduced and studied so-called nilpotent blocks. Using [25], we obtain the following result as an easy consequence of Theorem 1.2 .

Corollary 1.3. Let $k$ and $D$ be as in Theorem 1.2. Let $G$ be a finite group, and let $B$ be a nilpotent block of $k G$ with defect group $D$. Suppose $V$ is a finitely generated $B$-module with stable endomorphism ring $k$. Then the universal deformation ring $R(G, V)$ is isomorphic to $W[\mathbb{Z} / 2 \times \mathbb{Z} / 2]$.

The paper is organized as follows: In $₫ 2$, we provide some background on universal deformation rings for finite groups. In $\$ 3$, we study some subquotient modules of the free $k D$-module of rank 1 and describe lifts of two such $k D$-modules over $W$ using the ordinary irreducible representations of $D$. In \&4, we describe the locations of the indecomposable endo-trivial $k D$-modules in the stable Auslander-Reiten quiver of $k D$ using [3, 13]. In \$5, we complete the proof of Theorem 1.2 and Corollary 1.3.

The author would like to thank K. Erdmann and M. Linckelmann for helpful discussions on nilpotent blocks. She would also like to thank the referee for very useful comments which simplified some of the proofs.

\section{Preliminaries}

Let $k$ be an algebraically closed field of characteristic $p>0$, let $W$ be the ring of infinite Witt vectors over $k$ and let $F$ be the fraction field of $W$. Let $\mathcal{C}$ be the category of all complete local commutative Noetherian rings with residue field $k$. The morphisms in $\mathcal{C}$ are continuous $W$-algebra homomorphisms which induce the identity map on $k$.

Suppose $G$ is a finite group and $V$ is a finitely generated $k G$-module. If $R$ is an object in $\mathcal{C}$, a finitely generated $R G$-module $M$ is called a lift of $V$ over $R$ if $M$ is free over $R$ and $k \otimes_{R} M \cong V$ as $k G$-modules. Two lifts $M$ and $M^{\prime}$ of $V$ over $R$ are said to be isomorphic if there is an $R G$-module isomorphism $\alpha: M \rightarrow M^{\prime}$ which respects 
the $k G$-module isomorphisms $k \otimes_{R} M \cong V$ and $k \otimes_{R} M^{\prime} \cong V$. The isomorphism class of a lift of $V$ over $R$ is called a deformation of $V$ over $R$, and the set of such deformations is denoted by $\operatorname{Def}_{G}(V, R)$. The deformation functor $F_{V}: \mathcal{C} \rightarrow$ Sets is defined to be the covariant functor which sends an object $R$ in $\mathcal{C}$ to $\operatorname{Def}_{G}(V, R)$.

In case there exists an object $R(G, V)$ in $\mathcal{C}$ and a lift $U(G, V)$ of $V$ over $R(G, V)$ such that for each $R$ in $\mathcal{C}$ and for each lift $M$ of $V$ over $R$ there is a unique morphism $\alpha: R(G, V) \rightarrow R$ in $\mathcal{C}$ such that $M \cong R \otimes_{R(G, V), \alpha} U(G, V)$, then $R(G, V)$ is called the universal deformation ring of $V$ and the isomorphism class of the lift $U(G, V)$ is called the universal deformation of $V$. In other words, $R(G, V)$ represents the functor $F_{V}$ in the sense that $F_{V}$ is naturally isomorphic to $\operatorname{Hom}_{\mathcal{C}}(R(G, V),-)$. If $R(G, V)$ and the universal deformation corresponding to $U(G, V)$ exist, then they are unique up to unique isomorphism. For more information on deformation rings see [18] and 24.

The following four results were proved in [6] and in [5], respectively. Here $\Omega$ denotes the syzygy, or Heller, operator for $k G$ (see for example [1, §20]).

Proposition 2.1. [6, Prop. 2.1] Suppose $V$ is a finitely generated $k G$-module with stable endomorphism ring $\underline{\operatorname{End}}_{k G}(V)=k$. Then $V$ has a universal deformation ring $R(G, V)$.

Lemma 2.2. [6, Cors. 2.5 and 2.8] Let $V$ be a finitely generated $k G$-module with stable endomorphism ring $\underline{\operatorname{End}}_{k G}(V)=k$.

i. Then $\underline{\operatorname{End}}_{k G}(\Omega(V))=k$, and $R(G, V)$ and $R(G, \Omega(V))$ are isomorphic.

ii. There is a non-projective indecomposable $k G$-module $V_{0}$ (unique up to isomorphism) such that $\underline{\operatorname{End}}_{k G}\left(V_{0}\right)=k, V$ is isomorphic to $V_{0} \oplus P$ for some projective $k G$-module $P$, and $R(G, V)$ and $R\left(G, V_{0}\right)$ are isomorphic.

Lemma 2.3. [5, Lemma 2.3.2] Let $V$ be a finitely generated $k G$-module such that there is a non-split short exact sequence of $k G$-modules

$$
0 \rightarrow Y_{2} \rightarrow V \rightarrow Y_{1} \rightarrow 0
$$

with $\operatorname{Ext}_{k G}^{1}\left(Y_{1}, Y_{2}\right)=k$. Suppose that there exists a WG-module $X_{i}$ which is a lift of $Y_{i}$ over $W$ for $i=1,2$. Suppose further that

$$
\operatorname{dim}_{F} \operatorname{Hom}_{F G}\left(F \otimes_{W} X_{1}, F \otimes_{W} X_{2}\right)=\operatorname{dim}_{k} \operatorname{Hom}_{k G}\left(Y_{1}, Y_{2}\right)-1 .
$$

Then there exists a $W G$-module $X$ which is a lift of $V$ over $W$.

\section{The DIHEDRAL 2-GROUPS $D$}

Let $d \geq 3$ and let $D$ be a dihedral group of order $2^{d}$ given as

$$
D=\left\langle\sigma, \tau \mid \sigma^{2}=1=\tau^{2},(\sigma \tau)^{2^{d-2}}=(\tau \sigma)^{2^{d-2}}\right\rangle .
$$

Let $k$ be an algebraically closed field of characteristic $p=2$. The trivial simple $k D$-module $k$ is the unique irreducible $k D$-module up to isomorphism. The free $k D$ module $k D$ of rank one is indecomposable and its radical series has length $2^{d-1}+1$. The radical of $k D$ is generated as a $k D$-module by $(1+\sigma)$ and by $(1+\tau)$, and 
the socle of $k D$ is one-dimensional over $k$ and generated by $[(1+\sigma)(1+\tau)]^{2^{d-2}}=$ $[(1+\tau)(1+\sigma)]^{2^{d-2}}$. Hence

$$
\begin{aligned}
& \operatorname{rad}(k D)=k D(1+\sigma)+k D(1+\tau), \\
& \operatorname{soc}(k D)=k D(1+\sigma) \cap k D(1+\tau)=k[(1+\sigma)(1+\tau)]^{2^{d-2}} .
\end{aligned}
$$

From this description it follows that $\operatorname{rad}(k D) / \operatorname{soc}(k D)$ is isomorphic to the direct sum of two indecomposable $k D$-modules, namely

$$
\operatorname{rad}(k D) / \operatorname{soc}(k D) \cong k D(1+\sigma) / \operatorname{soc}(k D) \oplus k D(1+\tau) / \operatorname{soc}(k D) .
$$

Moreover, we have the following isomorphisms of $k D$-modules:

$$
\begin{aligned}
k D(1+\sigma) \cong k D \otimes_{k\langle\sigma\rangle} k & =\operatorname{Ind}_{\langle\sigma\rangle}^{D} k, \\
k D(1+\tau) \cong k D \otimes_{k\langle\tau\rangle} k & =\operatorname{Ind}_{\langle\tau\rangle}^{D} k .
\end{aligned}
$$

Let $\nu \in\{\sigma, \tau\}$, and define $E_{\nu}=k D(1+\nu) / \operatorname{soc}(k D)$. We have a commutative diagram of $k D$-modules of the form

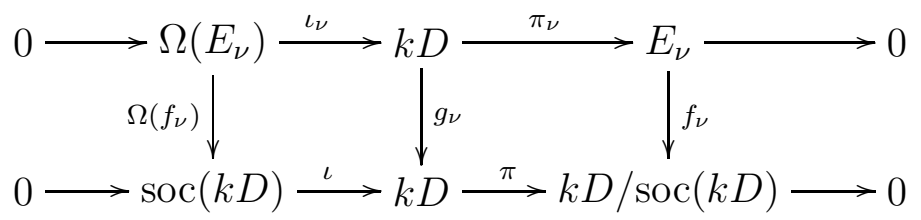

where $\pi_{\nu}(1)=(1+\nu)+\operatorname{soc}(k D), \pi(1)=1+\operatorname{soc}(k D), \iota_{\nu}$ and $\iota$ are inclusions, $g_{\nu}(1)=(1+\nu)$ and $f_{\nu}$ is induced by the inclusion map $k D(1+\nu) \hookrightarrow k D$. Since $f_{\nu}$ is injective, it follows that

$$
\operatorname{Ker}\left(\Omega\left(f_{\nu}\right)\right) \cong \operatorname{Ker}\left(g_{\nu}\right)=k D(1+\nu) .
$$

We now turn to representations of $D$ in characteristic 0 . Let $W$ be the ring of infinite Witt vectors over $k$, and let $F$ be the fraction field of $W$. Let $\zeta$ be a fixed primitive $2^{d-1}$-th root of unity in an algebraic closure of $F$. Then $D$ has $4+\left(2^{d-2}-1\right)$ ordinary irreducible characters $\chi_{1}, \chi_{2}, \chi_{3}, \chi_{4}, \chi_{5, i}, 1 \leq i \leq 2^{d-2}-1$, whose representations $\psi_{1}, \psi_{2}, \psi_{3}, \psi_{4}, \psi_{5, i}, 1 \leq i \leq 2^{d-2}-1$, are described in Table 1. In fact, the

TABLE 1 . The ordinary irreducible representations of $D$.

\begin{tabular}{|c||c|c|}
\hline & $\sigma$ & $\tau$ \\
\hline \hline$\psi_{1}=\chi_{1}$ & 1 & 1 \\
\hline$\psi_{2}=\chi_{2}$ & -1 & -1 \\
\hline$\psi_{3}=\chi_{3}$ & 1 & -1 \\
\hline$\psi_{4}=\chi_{4}$ & -1 & 1 \\
\hline$\psi_{5, i}$ & $\left(\begin{array}{cc}0 & 1 \\
1 & 0\end{array}\right)$ & $\left(\begin{array}{cc}0 & \zeta^{-i} \\
\zeta^{i} & 0\end{array}\right)$ \\
\hline
\end{tabular}

splitting field of $D$ is $F\left(\zeta+\zeta^{-1}\right)$, and the action of the Galois group $\operatorname{Gal}\left(F\left(\zeta+\zeta^{-1}\right) / F\right)$ on the ordinary irreducible characters divides the characters $\chi_{5, i}, i=1, \ldots, 2^{d-2}-1$, 
into $d-2$ Galois orbits $\mathcal{O}_{0}, \ldots, \mathcal{O}_{d-3}$ with $\mathcal{O}_{\ell}=\left\{\chi_{5,2^{d-3-\ell}(2 u-1)} \mid 1 \leq u \leq 2^{\ell}\right\}$ for $0 \leq \ell \leq d-3$. Since the Schur index over $F$ of each of the characters in these orbits is 1 , we obtain $d-2$ non-isomorphic simple $F D$-modules $V_{0}, \ldots, V_{d-3}$ whose characters $\rho_{0}, \ldots, \rho_{d-3}$ satisfy

$$
\rho_{\ell}=\sum_{u=1}^{2^{\ell}} \chi_{5,2^{d-3-\ell}(2 u-1)} \quad \text { for } 0 \leq \ell \leq d-3 .
$$

Moreover,

$$
\operatorname{End}_{F D}\left(V_{\ell}\right) \cong F\left(\zeta^{2^{d-3-\ell}}+\zeta^{-2^{d-3-\ell}}\right) \quad \text { for } 0 \leq \ell \leq d-3 .
$$

Lemma 3.1. Let

i. $(a, b) \in\{(1,3),(2,4)\}$, or

ii. $(a, b) \in\{(1,4),(2,3)\}$.

Let $N_{a, b}$ be an FD-module with character $\chi_{a}+\chi_{b}+\sum_{\ell=0}^{d-3} \rho_{\ell}$. Then there is a full $W D$-lattice $L_{a, b}$ in $N_{a, b}$ such that $L_{a, b} / 2 L_{a, b}$ is isomorphic to the $k D$-module $\operatorname{Ind}_{\langle\sigma\rangle}^{D} k$ in case $(i)$ and to the $k D$-module $\operatorname{Ind}_{\langle\tau\rangle}^{D} k$ in case $(i i)$.

Proof. Let $X_{\sigma}$ be the set of left cosets of $\langle\sigma\rangle$ in $D$, and let $W X_{\sigma}$ be the permutation module of $D$ over $W$ corresponding to $X_{\sigma}$. This means that $W X_{\sigma}$ is a free $W$-module with basis $\left\{m_{x} \mid x \in X_{\sigma}\right\}$ and $g \in D$ acts as $g \cdot m_{x}=m_{g x}$ for all $x \in X_{\sigma}$. Using the formula for the permutation character associated to the $F D$-module $F X_{\sigma}$, we see that this character is equal to $\chi_{1}+\chi_{3}+\sum_{\ell=0}^{d-3} \rho_{\ell}$.

There is a surjective $W D$-module homomorphism $h_{\sigma}: W D \rightarrow W X_{\sigma}$ which is defined by $h_{\sigma}(1)=m_{\langle\sigma\rangle}$. Then $\operatorname{Ker}\left(h_{\sigma}\right)=W D(1-\sigma)$ and we have a short exact sequence of $W D$-modules which are free over $W$

$$
0 \rightarrow W D(1-\sigma) \rightarrow W D \stackrel{h_{\sigma}}{\rightarrow} W X_{\sigma} \rightarrow 0 .
$$

Because the character of $F D$ is $\chi_{1}+\chi_{2}+\chi_{3}+\chi_{4}+2 \sum_{\ell=0}^{d-3} \rho_{\ell}$, the character of $F D(1-\sigma)$ must be $\chi_{2}+\chi_{4}+\sum_{\ell=0}^{d-3} \rho_{\ell}$. Tensoring (3.8) with $k$ over $W$, we obtain a short exact sequence of $k D$-modules

$$
0 \rightarrow k D(1-\sigma) \rightarrow k D \rightarrow k X_{\sigma} \rightarrow 0 \text {. }
$$

Since $k X_{\sigma} \cong \operatorname{Ind}_{\langle\sigma\rangle}^{D} k$ and the latter is isomorphic to $k D(1+\sigma)$ by (3.3) , Lemma 3.1 follows in case (i). Case (ii) is proved using the set $X_{\tau}$ of left cosets of $\langle\tau\rangle$ in $D$ instead.

\section{Endo-trivial Modules FOR $D$ in CHaraCteristic 2}

As before, let $k$ be an algebraically closed field of characteristic 2 . Since $D$ is a 2-group, it follows from [12] that the $k D$-modules $V$ with stable endomorphism ring $\underline{\operatorname{End}}_{k D}(V) \cong k$ are precisely the endo-trivial $k D$-modules, i.e. the $k D$-modules $V$ whose endomorphism ring over $k, \operatorname{End}_{k}(V)$, is as $k D$-module stably isomorphic to the trivial $k D$-module $k$. The latter modules have been completely classified in [13] (see also [14]). We will use this description to determine the location of the 
indecomposable endo-trivial $k D$-modules in the stable Auslander-Reiten quiver of $k D$.

Remark 4.1. Let $z=(\sigma \tau)^{2^{d-2}}$ be the involution in the center of $D$. The poset of all elementary abelian subgroups of $D$ of rank at least 2 consists of two conjugacy classes of maximal elementary abelian subgroups of rank exactly 2 . These conjugacy classes are represented by $K_{1}=\langle\sigma, z\rangle$ and $K_{2}=\langle\tau, z\rangle$. Let $T(D)$ denote the group of equivalence classes of endo-trivial $k D$-modules as in [13]. Consider the map

$$
\Xi: T(D) \rightarrow \mathbb{Z} \times \mathbb{Z}
$$

defined by $\Xi([M])=\left(a_{1}, a_{2}\right)$ when $\operatorname{Res}_{K_{i}}^{D} M \cong \Omega_{K_{i}}^{a_{i}}(k) \oplus F_{M, i}$ for some free $k K_{i}$-module $F_{M, i}$ for $i=1,2$. In particular, $\Xi([k])=(0,0)$ and $\Xi\left(\left[\Omega^{m}(M)\right]\right)=\Xi([M])+(m, m)$ for all endo-trivial $k D$-modules $M$ and all integers $m$. By [13, Thm. 5.4], $\Xi$ is injective and the image of $\Xi$ is generated by $(1,1)$ and $(1,-1)$ (and also by $(1,1)$ and $(-1,1)$ ).

As in 93 , let $E_{\sigma}=k D(1+\sigma) / \operatorname{soc}(k D)$ and let $E_{\tau}=k D(1+\tau) / \operatorname{soc}(k D)$. By (3.2), $\operatorname{rad}(k D) / \operatorname{soc}(k D) \cong E_{\sigma} \oplus E_{\tau}$. The almost split sequence ending in $\Omega^{-1}(k)=$ $k D / \operatorname{soc}(k D)$ has thus the form

$$
0 \rightarrow \Omega(k) \rightarrow k D \oplus E_{\sigma} \oplus E_{\tau} \stackrel{\mu_{-1}}{\longrightarrow} \Omega^{-1}(k) \rightarrow 0
$$

where $\left.\mu_{-1}\right|_{E_{\nu}}$ is the rightmost vertical homomorphism $f_{\nu}$ in the diagram (3.4) and $\left.\mu_{-1}\right|_{k D}$ is the natural projection. It follows for example from [3, Lemma 5.4] that $E_{\sigma}$ and $E_{\tau}$ are endo-trivial. Moreover, $\Xi\left(\left[E_{\sigma}\right]\right)=(1,-1)$ and $\Xi\left(\left[E_{\tau}\right]\right)=(-1,1)$. In particular, $T(D)$ is generated by $[\Omega(k)]$ and $\left[E_{\sigma}\right]$ (and also by $[\Omega(k)]$ and $\left[E_{\tau}\right]$ ).

Let $\nu \in\{\sigma, \tau\}$, and define $A_{\nu, 0}=k$ and $A_{\nu, 1}=\Omega\left(E_{\nu}\right)$. For $n \geq 2$, define $A_{\nu, n}$ to be the unique indecomposable $k D$-module, up to isomorphism, in the equivalence class of the endo-trivial $k D$-module $A_{\nu, 1} \otimes_{k} A_{\nu, n-1}$. Then the trivial simple $k D$-module $k=A_{\sigma, 0}=A_{\tau, 0}$ together with the $k D$-modules $A_{\sigma, n}, A_{\tau, n}$ for $n \geq 1$ give a complete set of representatives of the $\Omega$-orbits of the indecomposable endo-trivial $k D$-modules. We have $\Xi\left(\left[A_{\sigma, n}\right]\right)=(2 n, 0)$ and $\Xi\left(\left[A_{\tau, n}\right]\right)=(0,2 n)$ for all $n \geq 0$.

Lemma 4.2. The finitely generated indecomposable endo-trivial $k D$-modules are exactly the modules in the two components of the stable Auslander-Reiten quiver of $k D$ containing the trivial simple $k D$-module $k$ and $\Omega(k)$.

More precisely, let $A_{\sigma, n}$ and $A_{\tau, n}$ be as in Remark 4.1 for $n \geq 0$. Then the almost split sequence ending in $k$ has the form

$$
0 \rightarrow \Omega^{2}(k) \rightarrow A_{\sigma, 1} \oplus A_{\tau, 1} \stackrel{\mu_{1}}{\rightarrow} k \rightarrow 0 .
$$

Let $\nu \in\{\sigma, \tau\}$ and let $n \geq 1$. Then the almost split sequence ending in $A_{\nu, n}$ has the form

$$
0 \rightarrow \Omega^{2}\left(A_{\nu, n}\right) \rightarrow A_{\nu, n+1} \oplus \Omega^{2}\left(A_{\nu, n-1}\right) \stackrel{\mu_{\nu, n+1}}{\longrightarrow} A_{\nu, n} \rightarrow 0 .
$$

Proof. Since $\Omega$ defines an equivalence of the stable module category of finitely generated $k D$-modules with itself, we can apply $\Omega$ to the almost split sequence (4.2) to obtain the almost split sequence ending in $k$ up to free direct summands of the middle term. Since the sequence (4.2) is the only almost split sequence having $k D$ as a summand of the middle term, the almost split sequence ending in $k$ is as in (4.3). 
Given an indecomposable $k D$-module $M$ of odd $k$-dimension, it follows from 3 , Thm. 3.6 and Cor. 4.7] that

$$
0 \rightarrow \Omega^{2}(k) \otimes_{k} M \rightarrow\left(A_{\sigma, 1} \otimes_{k} M\right) \oplus\left(A_{\tau, 1} \otimes_{k} M\right) \rightarrow M \rightarrow 0
$$

is the almost split sequence ending in $M$ modulo projective direct summands. Since all endo-trivial $k D$-modules have odd $k$-dimension, we can apply the sequence (4.5) to $M=A_{\nu, n}$ for $\nu \in\{\sigma, \tau\}$ and all $n \geq 1$. This means that modulo free direct summands the almost split sequence ending in $A_{\nu, n}$ has the form

$$
0 \rightarrow \Omega^{2}(k) \otimes_{k} A_{\nu, n} \rightarrow\left(A_{\sigma, 1} \otimes_{k} A_{\nu, n}\right) \oplus\left(A_{\tau, 1} \otimes_{k} A_{\nu, n}\right) \rightarrow A_{\nu, n} \rightarrow 0 .
$$

Note that $\Xi\left(\left[A_{\nu^{\prime}, 1} \otimes_{k} A_{\nu, n}\right]\right)=(2,2)+\Xi\left(\left[A_{\nu, n-1}\right]\right)=\Xi\left(\left[\Omega^{2}\left(A_{\nu, n-1}\right)\right]\right)$ if $\left\{\nu, \nu^{\prime}\right\}=\{\sigma, \tau\}$. Since the sequence (4.2) is the only almost split sequence having $k D$ as a summand of the middle term, it follows that the almost split sequence ending in $A_{\nu, n}$ is as in (4.4). This completes the proof of Lemma 4.2.

Lemma 4.3. Let $\nu \in\{\sigma, \tau\}$, let $n \geq 0$ and let $A_{\nu, n}$ be as in Remark 4.1. Then $\operatorname{dim}_{k} A_{\nu, n}=n 2^{d-1}+1$ and $\operatorname{Res}_{C}^{D} A_{\nu, n} \cong k \oplus(k C)^{n 2^{d-2}}$ for $C \in\{\langle\sigma\rangle,\langle\tau\rangle\}$. Moreover, there is a short exact sequence of $k D$-modules

$$
0 \rightarrow \operatorname{Ind}_{\langle\nu\rangle}^{D} k \rightarrow A_{\nu, n+1} \rightarrow A_{\nu, n} \rightarrow 0 .
$$

Proof. When we restrict the almost split sequences (4.3) and (4.4) to the elementary abelian subgroups $K_{1}$ and $K_{2}$ from Remark 4.1, it follows that the resulting short exact sequences of $k K_{i}$-modules split for $i=1,2$. Define $\phi_{\nu, 1}: A_{\nu, 1} \rightarrow k$ to be the restriction of the homomorphism $\mu_{1}$ in (4.3) to the component $A_{\nu, 1}$, and for $n \geq 1$ define $\phi_{\nu, n+1}: A_{\nu, n+1} \rightarrow A_{\nu, n}$ to be the restriction of the homomorphism $\mu_{\nu, n+1}$ in (4.4) to the component $A_{\nu, n+1}$. For $n \geq 1$, let $\Phi_{\nu, n}: A_{\nu, n} \rightarrow k$ be the composition $\Phi_{\nu, n}=\phi_{\nu, 1} \circ \phi_{\nu, 2} \circ \ldots \circ \phi_{\nu, n}$. Then it follows that the homomorphism $\Phi_{n}: A_{\sigma, n} \oplus A_{\tau, n} \rightarrow k$, which restricted to $A_{\nu, n}$ is given by $\Phi_{\nu, n}$, splits when viewed as a homomorphism of $k K_{i}$-modules for $i=1,2$. Since $\Xi\left(\left[A_{\sigma, n}\right]\right)=(2 n, 0)$ and $\Xi\left(\left[A_{\tau, n}\right]\right)=(0,2 n)$, this implies that for all $n \geq 1$ we have a short exact sequence of $k D$-modules of the form

$$
0 \rightarrow \Omega^{2 n}(k) \rightarrow A_{\sigma, n} \oplus A_{\tau, n} \stackrel{\Phi_{n}}{\longrightarrow} k \rightarrow 0 .
$$

Inductively, we see that $\Omega^{2 n}(k)$ has $k$-dimension $n 2^{d}+1$. Because the $k$-dimensions of $A_{\sigma, n}$ and $A_{\tau, n}$ coincide, this implies that $\operatorname{dim}_{k} A_{\nu, n}=\frac{1}{2}\left(n 2^{d}+2\right)=n 2^{d-1}+1$.

Let $C \in\{\langle\sigma\rangle,\langle\tau\rangle\}$. Since $E_{\nu}=k D(1+\nu) / \operatorname{soc}(k D)$, it follows that $\operatorname{Res}_{C}^{D} E_{\nu}$ is stably isomorphic to $k$. Hence we obtain for $A_{\nu, 1}=\Omega\left(E_{\nu}\right)$ that $\operatorname{Res}_{C}^{D} A_{\nu, 1}$ also is stably isomorphic to $k$. Therefore, it follows by induction from the almost split sequence (4.4) that $\operatorname{Res}_{C}^{D} A_{\nu, n+1}$ is stably isomorphic to $k$ for all $n \geq 1$. Comparing $k$-dimensions it follows that $\operatorname{Res}_{C}^{D} A_{\nu, n} \cong k \oplus(k C)^{n 2^{d-2}}$ for all $n \geq 0$.

To construct a short exact sequence of the form (4.7), recall that the almost split sequence (4.3) is obtained by applying $\Omega$ to the almost split sequence (4.2). Since the restriction of the homomorphism $\mu_{-1}$ in (4.2) to the component $E_{\nu}$ is the same as the homomorphism $f_{\nu}$ in the diagram (3.4), the restriction of the homomorphism $\mu_{1}$ in (4.3) to the component $A_{\nu, 1}$ is the same as $\Omega\left(f_{\nu}\right)$. By (3.5) we have $\operatorname{Ker}\left(\Omega\left(f_{\nu}\right)\right) \cong$ 
$k D(1+\nu)$, which implies by (3.3) that there is a short exact sequence of $k D$-modules of the form

$$
0 \rightarrow \operatorname{Ind}_{\langle\nu\rangle}^{D} k \rightarrow A_{\nu, 1} \rightarrow k \rightarrow 0 .
$$

Now let $n \geq 1$. Tensoring the sequence (4.8) with $A_{\nu, n}$, we obtain a short exact sequence of $k D$-modules of the form

$$
0 \rightarrow\left(\operatorname{Ind}_{\langle\nu\rangle}^{D} k\right) \otimes_{k} A_{\nu, n} \rightarrow A_{\nu, 1} \otimes_{k} A_{\nu, n} \rightarrow A_{\nu, n} \rightarrow 0 .
$$

Since $\left(\operatorname{Ind}_{\langle\nu\rangle}^{D} k\right) \otimes_{k} A_{\nu, n} \cong \operatorname{Ind}_{\langle\nu\rangle}^{D}\left(\operatorname{Res}_{\langle\nu\rangle}^{D} A_{\nu, n}\right)$ and $\operatorname{Res}_{\langle\nu\rangle}^{D} A_{\nu, n} \cong k \oplus(k\langle\nu\rangle)^{n 2^{d-2}}$, it follows that

$$
\left(\operatorname{Ind}_{\langle\nu\rangle}^{D} k\right) \otimes_{k} A_{\nu, n} \cong \operatorname{Ind}_{\langle\nu\rangle}^{D} k \oplus(k D)^{n 2^{d-2}} .
$$

By definition, $A_{\nu, n+1}$ is the unique indecomposable $k D$-module, up to isomorphism, in the equivalence class of the endo-trivial $k D$-module $A_{\nu, 1} \otimes_{k} A_{\nu, n}$. Comparing $k$ dimensions it follows that $\operatorname{dim}_{k} A_{\nu, 1} \otimes_{k} A_{\nu, n}=n 2^{d-2} \cdot 2^{d}+\operatorname{dim}_{k} A_{\nu, n+1}$. Hence

$$
A_{\nu, 1} \otimes_{k} A_{\nu, n} \cong A_{\nu, n+1} \oplus(k D)^{n 2^{d-2}} .
$$

By splitting off the free $k D$-module $(k D)^{n 2^{d-2}}$ from the first and second term of the short exact sequence (4.9) we obtain a short exact sequence of $k D$-modules of the form (4.7).

\section{UNIVERSAL DEFORMATION RINGS FOR $D$ AND NILPOTENT BLOCKS}

In this section, we prove Theorem 1.2 and Corollary 1.3. We use the notations of the previous sections. In particular, $k$ is an algebraically closed field of characteristic 2, $W$ is the ring of infinite Witt vectors over $k$ and $F$ is the fraction field of $W$.

For $\nu \in\{\sigma, \tau\}$ and $n \geq 0$, let $A_{\nu, n}$ be as in Remark 4.1. We first analyze all extensions of $A_{\nu, n}$ by $A_{\nu, n}$ by using the extensions of $k$ by $k$ which are described in the following remark.

Remark 5.1. Suppose $N$ is a $k D$-module which lies in a non-split short exact sequence of $k D$-modules

$$
0 \rightarrow k \rightarrow N \rightarrow k \rightarrow 0 .
$$

Then $N$ is isomorphic to $N_{\lambda}$ for some $\lambda \in k^{*} \cup\{\sigma, \tau\}$ where a representation $\varphi_{\lambda}$ of $N_{\lambda}$ is given by the following $2 \times 2$ matrices over $k$.
a. If $\lambda \in k^{*}$ then $\varphi_{\lambda}(\sigma)=\left(\begin{array}{cc}1 & 1 \\ 0 & 1\end{array}\right), \varphi_{\lambda}(\tau)=\left(\begin{array}{ll}1 & \lambda \\ 0 & 1\end{array}\right)$.
b. If $\left\{\lambda, \lambda^{\prime}\right\}=\{\sigma, \tau\}$ then $\varphi_{\lambda}(\lambda)=\left(\begin{array}{ll}1 & 1 \\ 0 & 1\end{array}\right), \varphi_{\lambda}\left(\lambda^{\prime}\right)=\left(\begin{array}{ll}1 & 0 \\ 0 & 1\end{array}\right)$.

We obtain the following restrictions of $N_{\lambda}$ to the subgroups $\langle\sigma\rangle$ and $\langle\tau\rangle$ of $D$.

- In case (a), $\operatorname{Res}_{C}^{D} N_{\lambda} \cong k C$ for $C \in\{\langle\sigma\rangle,\langle\tau\rangle\}$.

- In case (b), $\operatorname{Res}_{\langle\lambda\rangle}^{D} N_{\lambda} \cong k\langle\lambda\rangle$ and $\operatorname{Res}_{\left\langle\lambda^{\prime}\right\rangle}^{D} N_{\lambda} \cong k^{2}$. 
Lemma 5.2. Let $\nu \in\{\sigma, \tau\}$, let $n \geq 0$, and let $A_{\nu, n}$ be as in Remark 4.1. Then $\operatorname{Ext}_{k D}^{1}\left(A_{\nu, n}, A_{\nu, n}\right) \cong k^{2}$. Suppose $Z_{\nu, n}$ is a $k D$-module which lies in a non-split short exact sequence of $k D$-modules

$$
0 \rightarrow A_{\nu, n} \rightarrow Z_{\nu, n} \rightarrow A_{\nu, n} \rightarrow 0 .
$$

Then $Z_{\nu, n}$ is isomorphic to $B_{\nu, n, \lambda}=A_{\nu, n} \otimes_{k} N_{\lambda}$ for some $\lambda \in k^{*} \cup\{\sigma, \tau\}$ where $N_{\lambda}$ is as in Remark 5.1. Let $\left\{\nu, \nu^{\prime}\right\}=\{\sigma, \tau\}$.

i. If $\lambda \in k^{*} \cup\{\nu\}$ then $B_{\nu, n, \lambda} \cong N_{\lambda} \oplus(k D)^{n}$.

ii. If $\lambda=\nu^{\prime}$ then $B_{\nu, 0, \nu^{\prime}} \cong N_{\nu^{\prime}}$ and for $n \geq 1, B_{\nu, n, \nu^{\prime}}$ is a non-split extension of $B_{\nu, n-1, \nu^{\prime}}$ by $k D(1+\nu) \oplus k D(1+\nu)$. Moreover, $\operatorname{Res}_{\left\langle\nu^{\prime}\right\rangle}^{D} B_{\nu, n, \nu^{\prime}} \cong\left(k\left\langle\nu^{\prime}\right\rangle\right)^{n 2^{d-1}+1}$ and $\operatorname{Res}_{\langle\nu\rangle}^{D} B_{\nu, n, \nu^{\prime}} \cong k^{2} \oplus(k\langle\nu\rangle)^{n 2^{d-1}}$.

Proof. Since $A_{\nu, n}$ is endo-trivial, it follows from [3, Thm. 2.6] that the natural homomorphism

$$
\operatorname{Ext}_{k D}^{1}(k, k) \rightarrow \operatorname{Ext}_{k D}^{1}\left(A_{\nu, n}, A_{\nu, n}\right)
$$

resulting from tensoring short exact sequences of the form (5.1) with $A_{\nu, n}$ is a monomorphism. Moreover,

$$
\operatorname{Ext}_{k D}^{1}\left(A_{\nu, n}, A_{\nu, n}\right) \cong \mathrm{H}^{1}\left(D, A_{\nu, n}^{*} \otimes_{k} A_{\nu, n}\right) \cong \mathrm{H}^{1}(D, k) \cong \operatorname{Ext}_{k D}^{1}(k, k) \cong k^{2}
$$

where the second isomorphism follows since $A_{\nu, n}$ is endo-trivial. Hence the homomorphism in (5.3) is an isomorphism, which means that we only need to prove the descriptions of $B_{\nu, n, \lambda}=A_{\nu, n} \otimes_{k} N_{\lambda}$ given in (i) and (ii) of the statement of Lemma 5.2. For $n=0$ this follows from Remark 5.1 since $A_{\nu, 0}=k$ and so $B_{\nu, 0, \lambda}=N_{\lambda}$.

By Lemma 4.3, there is a short exact sequence of $k D$-modules of the form (4.7). Tensoring this sequence with $N_{\lambda}$ over $k$ gives a short exact sequence of $k D$-modules of the form

$$
0 \rightarrow\left(\operatorname{Ind}_{\langle\nu\rangle}^{D} k\right) \otimes_{k} N_{\lambda} \rightarrow B_{\nu, n+1, \lambda} \rightarrow B_{\nu, n, \lambda} \rightarrow 0
$$

where $\left(\operatorname{Ind}_{\langle\nu\rangle}^{D} k\right) \otimes_{k} N_{\lambda} \cong \operatorname{Ind}_{\langle\nu\rangle}^{D}\left(\operatorname{Res}_{\langle\nu\rangle}^{D} N_{\lambda}\right)$.

Let first $\lambda \in k^{*} \cup\{\nu\}$. Then $\operatorname{Res}_{\langle\nu\rangle}^{D} N_{\lambda} \cong k\langle\nu\rangle$ and hence $\operatorname{Ind}_{\langle\nu\rangle}^{D}\left(\operatorname{Res}_{\langle\nu\rangle}^{D} N_{\lambda}\right) \cong k D$. It follows by induction that $B_{\nu, n+1, \lambda} \cong N_{\lambda} \oplus(k D)^{n+1}$, which proves (i).

Now let $\lambda=\nu^{\prime}$. Then $\operatorname{Res}_{\langle\nu\rangle}^{D} N_{\nu^{\prime}} \cong k^{2}$ and hence

$$
\operatorname{Ind}_{\langle\nu\rangle}^{D}\left(\operatorname{Res}_{\langle\nu\rangle}^{D} N_{\nu}^{\prime}\right) \cong \operatorname{Ind}_{\langle\nu\rangle}^{D} k^{2} \cong k D(1+\nu) \oplus k D(1+\nu) .
$$

Thus $B_{\nu, n+1, \nu^{\prime}}$ is an extension of $B_{\nu, n, \nu^{\prime}}$ by $k D(1+\nu) \oplus k D(1+\nu)$. Since $\operatorname{Res}_{\left\langle\nu^{\prime}\right\rangle}^{D} k D(1+$ $\nu) \cong\left(k\left\langle\nu^{\prime}\right\rangle\right)^{2^{d-2}}$, it follows by induction that $\operatorname{Res}_{\left\langle\nu^{\prime}\right\rangle}^{D} B_{\nu, n+1, \nu^{\prime}} \cong\left(k\left\langle\nu^{\prime}\right\rangle\right)^{(n+1) 2^{d-1}+1}$. On the other hand, $\operatorname{Res}_{\langle\nu\rangle}^{D} k D(1+\nu) \cong k^{2} \oplus(k\langle\nu\rangle)^{2^{d-2}-1}$. Hence the restriction of the left term in the short exact sequence (5.4) to $\langle\nu\rangle$ is isomorphic to $k^{4} \oplus(k\langle\nu\rangle)^{2^{d-1}-2}$. Thus by induction, $\operatorname{Res}_{\langle\nu\rangle}^{D} B_{\nu, n+1, \nu^{\prime}}$ lies in a short exact sequence of $k\langle\nu\rangle$-modules of the form

$$
0 \rightarrow k^{4} \oplus(k\langle\nu\rangle)^{2^{d-1}-2} \rightarrow \operatorname{Res}_{\langle\nu\rangle}^{D} B_{\nu, n+1, \nu^{\prime}} \rightarrow k^{2} \oplus(k\langle\nu\rangle)^{n 2^{d-1}} \rightarrow 0 .
$$


Since $\operatorname{Res}_{\langle\nu\rangle}^{D} B_{\nu, n+1, \nu^{\prime}}$ is an extension of $\operatorname{Res}_{\langle\nu\rangle}^{D} A_{\nu, n+1}$ by itself and since $\operatorname{Res}_{\langle\nu\rangle}^{D} A_{\nu, n+1} \cong$ $k \oplus(k\langle\nu\rangle)^{(n+1) 2^{d-2}}$ by Lemma 4.3, it follows that $\operatorname{Res}_{\langle\nu\rangle}^{D} B_{\nu, n+1, \nu^{\prime}} \cong k^{2} \oplus(k\langle\nu\rangle)^{(n+1) 2^{d-1}}$. In particular, the sequence (5.4) does not split when $\lambda=\nu^{\prime}$, since it does not split when restricted to $\langle\nu\rangle$. This proves (ii).

The next result uses the restrictions of $A_{\nu, n}$ and $B_{\nu, n, \lambda}$ to the cyclic subgroups $\langle\sigma\rangle$ and $\langle\tau\rangle$ of $D$.

Lemma 5.3. Let $\nu \in\{\sigma, \tau\}$, let $n \geq 0$, and let $A_{\nu, n}$ be as in Remark 4.1. Then there is a surjective $W$-algebra homomorphism $\alpha: W[\mathbb{Z} / 2 \times \mathbb{Z} / 2] \rightarrow R\left(D, A_{\nu, n}\right)$ in $\mathcal{C}$.

Proof. Let $R=R\left(D, A_{\nu, n}\right)$ and let $U_{\nu, n}$ be a universal lift of $A_{\nu, n}$ over $R$. Let $C_{\sigma}=\langle\sigma\rangle$ and $C_{\tau}=\langle\tau\rangle$, and let $C \in\left\{C_{\sigma}, C_{\tau}\right\}$. By Lemma 4.3, $\operatorname{Res}_{C}^{D} A_{\nu, n} \cong k \oplus(k C)^{n 2^{d-2}}$. In particular, $\operatorname{Res}_{C}^{D} A_{\nu, n}$ is a $k C$-module with stable endomorphism ring $k$, and hence it has a universal deformation ring. Moreover, $R\left(C, \operatorname{Res}_{C}^{D} A_{\nu, n}\right) \cong W[\mathbb{Z} / 2]$. Let $U_{\nu, n, C}$ be a universal lift of $\operatorname{Res}_{C}^{D} A_{\nu, n}$ over $W[\mathbb{Z} / 2]$. Then there exists a unique $W$-algebra homomorphism $\alpha_{C}: W[\mathbb{Z} / 2] \rightarrow R$ in $\mathcal{C}$ such that $\operatorname{Res}_{C}^{D} U_{\nu, n} \cong R \otimes_{W[\mathbb{Z} / 2], \alpha_{C}} U_{\nu, n, C}$. Since the completed tensor product over $W$ is the coproduct in the category $\mathcal{C}$, we get a $W$-algebra homomorphism

$$
\alpha=\alpha_{C_{\sigma}} \otimes \alpha_{C_{\tau}}: W[\mathbb{Z} / 2] \otimes_{W} W[\mathbb{Z} / 2] \rightarrow R
$$

in $\mathcal{C}$ with $\alpha\left(x_{1} \otimes x_{2}\right)=\alpha_{C_{\sigma}}\left(x_{1}\right) \alpha_{C_{\tau}}\left(x_{2}\right)$.

By Lemma [5.2, every non-trivial lift of $A_{\nu, n}$ over the dual numbers $k[\epsilon] /\left(\epsilon^{2}\right)$ is as $k D$-module isomorphic to $B_{\nu, n, \lambda}$ for some $\lambda \in k^{*} \cup\{\sigma, \tau\}$. The description of these modules shows that their restrictions to $C_{\sigma}$ and $C_{\tau}$ are as follows:

i. If $\lambda \in k^{*}$ then $\operatorname{Res}_{C}^{D} B_{\nu, n, \lambda}=(k C)^{n 2^{d-1}+1}$ for $C \in\left\{C_{\sigma}, C_{\tau}\right\}$.

ii. If $\left\{\lambda, \lambda^{\prime}\right\}=\{\sigma, \tau\}$, then $\operatorname{Res}_{C_{\lambda}}^{D} B_{\nu, n, \lambda}=\left(k C_{\lambda}\right)^{n 2^{d-1}+1}$ and $\operatorname{Res}_{C_{\lambda^{\prime}}}^{D} B_{\nu, n, \lambda}=$ $k^{2} \oplus\left(k C_{\lambda^{\prime}}\right)^{n 2^{d-1}}$.

Note that for $C \in\left\{C_{\sigma}, C_{\tau}\right\}, k^{2} \oplus(k C)^{n 2^{d-1}}$ corresponds to the trivial lift of $\operatorname{Res}_{C}^{D} A_{\nu, n}$ over $k[\epsilon] /\left(\epsilon^{2}\right)$, and $(k C)^{n 2^{d-1}+1}$ corresponds to a non-trivial lift of $\operatorname{Res}_{C}^{D} A_{\nu, n}$ over $k[\epsilon] /\left(\epsilon^{2}\right)$.

Let $\lambda \in k^{*} \cup\{\sigma, \tau\}$, and let $f_{\lambda}: R \rightarrow k[\epsilon] /\left(\epsilon^{2}\right)$ be a morphism corresponding to a non-trivial lift of $A_{\nu, n}$ over $k[\epsilon] /\left(\epsilon^{2}\right)$ with underlying $k D$-module structure given by $B_{\nu, n, \lambda}$. Then $f_{\lambda} \circ \alpha=f_{\lambda} \circ\left(\alpha_{C_{\sigma}} \otimes \alpha_{C_{\tau}}\right)$ corresponds to the pair of lifts of the pair $\left(\operatorname{Res}_{C_{\sigma}}^{D} A_{\nu, n}, \operatorname{Res}_{C_{\tau}}^{D} A_{\nu, n}\right)$ over $k[\epsilon] /\left(\epsilon^{2}\right)$ with underlying $k D$-module structure given by the pair ( $\left.\operatorname{Res}_{C_{\sigma}}^{D} B_{\nu, n, \lambda}, \operatorname{Res}_{C_{\tau}}^{D} B_{\nu, n, \lambda}\right)$. Hence (i) and (ii) above imply that if $f$ runs through the morphisms $R \rightarrow k[\epsilon] /\left(\epsilon^{2}\right)$, then $f \circ \alpha$ runs through the morphisms $W[\mathbb{Z} / 2] \otimes_{W} W[\mathbb{Z} / 2] \rightarrow k[\epsilon] /\left(\epsilon^{2}\right)$. This implies $\alpha$ is surjective.

We next determine how many non-isomorphic lifts $A_{\nu, n}$ has over $W$.

Lemma 5.4. Let $\nu \in\{\sigma, \tau\}$, let $n \geq 0$, and let $A_{\nu, n}$ be as in Remark 4.1. Then $A_{\nu, n}$ has four pairwise non-isomorphic lifts over $W$ corresponding to four distinct morphisms $R\left(D, A_{\nu, n}\right) \rightarrow W$ in $\mathcal{C}$. 
Proof. We use Lemmas 2.3 and 3.1 to prove this. If $n=0$ then $A_{\nu, 0}$ is the trivial simple $k D$-module $k$ which has four pairwise non-isomorphic lifts over $W$ whose $F$-characters are given by the four ordinary irreducible characters of degree one $\chi_{1}, \chi_{2}, \chi_{3}, \chi_{4}$ (see Table 1). By Lemma 4.3, there is a short exact sequence of $k D$ modules of the form

$$
0 \rightarrow \operatorname{Ind}_{\langle\nu\rangle}^{D} k \rightarrow A_{\nu, n+1} \rightarrow A_{\nu, n} \rightarrow 0 .
$$

By Frobenius reciprocity and the Eckman-Shapiro Lemma, we have for all $n \geq 0$

$$
\begin{aligned}
& \operatorname{Hom}_{k D}\left(A_{\nu, n}, \operatorname{Ind}_{\langle\nu\rangle}^{D} k\right) \cong \operatorname{Hom}_{k\langle\nu\rangle}\left(\operatorname{Res}_{\langle\nu\rangle}^{D} A_{\nu, n}, k\right) \cong k^{n 2^{d-2}+1} \text { and } \\
& \operatorname{Ext}_{k D}^{1}\left(A_{\nu, n}, \operatorname{Ind}_{\langle\nu\rangle}^{D} k\right) \cong \operatorname{Ext}_{k\langle\nu\rangle}^{1}\left(\operatorname{Res}_{\langle\nu\rangle}^{D} A_{\nu, n}, k\right) \cong k
\end{aligned}
$$

where the second isomorphisms follow since $\operatorname{Res}_{\langle\nu\rangle}^{D} A_{\nu, n} \cong k \oplus(k\langle\nu\rangle)^{n 2^{d-2}}$ by Lemma 4.3 .

Let $\rho=\sum_{\ell=0}^{d-3} \rho_{\ell}$ for $\rho_{\ell}$ as in (3.6). Then every $F D$-module $T$ with $F$-character $\rho$ satisfies by (3.7)

$$
\operatorname{dim}_{F} \operatorname{End}_{F D}(T)=\sum_{\ell=0}^{d-3} \operatorname{dim}_{F} \operatorname{End}_{F D}\left(V_{\ell}\right)=\sum_{\ell=0}^{d-3} 2^{\ell}=2^{d-2}-1 .
$$

Let $(c, d)=(3,4)$ if $\nu=\sigma$, and let $(c, d)=(4,3)$ if $\nu=\tau$. Assume by induction that $A_{\nu, n}$ has four pairwise non-isomorphic lifts over $W$ whose $F$-characters are given by

i. $\chi_{1}+m\left(\chi_{1}+\chi_{c}\right)+m\left(\chi_{2}+\chi_{d}\right)+2 m \rho$, or $\chi_{c}+m\left(\chi_{1}+\chi_{c}\right)+m\left(\chi_{2}+\chi_{d}\right)+2 m \rho$, or

ii. $\chi_{2}+m\left(\chi_{1}+\chi_{c}\right)+m\left(\chi_{2}+\chi_{d}\right)+2 m \rho$, or $\chi_{d}+m\left(\chi_{1}+\chi_{c}\right)+m\left(\chi_{2}+\chi_{d}\right)+2 m \rho$

if $n=2 m$ for some $m \geq 0$, and by

$$
\begin{aligned}
\text { i'. } & \chi_{1}+m\left(\chi_{1}+\chi_{c}\right)+(m+1)\left(\chi_{2}+\chi_{d}\right)+(2 m+1) \rho, \text { or } \\
& \chi_{c}+m\left(\chi_{1}+\chi_{c}\right)+(m+1)\left(\chi_{2}+\chi_{d}\right)+(2 m+1) \rho, \text { or } \\
\text { ii'. } & \chi_{2}+(m+1)\left(\chi_{1}+\chi_{c}\right)+m\left(\chi_{2}+\chi_{d}\right)+(2 m+1) \rho, \text { or } \\
& \chi_{d}+(m+1)\left(\chi_{1}+\chi_{c}\right)+m\left(\chi_{2}+\chi_{d}\right)+(2 m+1) \rho
\end{aligned}
$$

if $n=2 m+1$ for some $m \geq 0$. By Lemma 3.1. $\operatorname{Ind}_{\langle\nu\rangle}^{D} k$ has two pairwise nonisomorphic lifts over $W$ with $F$-characters $\chi_{1}+\chi_{c}+\rho$ or $\chi_{2}+\chi_{d}+\rho$. If $\mathcal{A}_{\nu, n}$ is a lift of $A_{\nu, n}$ over $W$ with $F$-character as in (i) or (ii'), let $\mathcal{I}$ be a lift of $\operatorname{Ind}_{\langle\nu\rangle}^{D} k$ over $W$ with $F$-character $\chi_{2}+\chi_{d}+\rho$. If $\mathcal{A}_{\nu, n}$ is a lift of $A_{\nu, n}$ over $W$ with $F$-character as in (ii) or (i'), let $\mathcal{I}$ be a lift of $\operatorname{Ind}_{\langle\nu\rangle}^{D} k$ over $W$ with $F$-character $\chi_{1}+\chi_{c}+\rho$. Then we obtain for $n=2 m$,

$$
\operatorname{dim}_{F} \operatorname{Hom}_{F D}\left(F \otimes_{W} \mathcal{A}_{\nu, n}, F \otimes_{W} \mathcal{I}\right)=2 m 2^{d-2}=n 2^{d-2},
$$

and for $n=2 m+1$,

$$
\operatorname{dim}_{F} \operatorname{Hom}_{F D}\left(F \otimes_{W} \mathcal{A}_{\nu, n}, F \otimes_{W} \mathcal{I}\right)=(2 m+1) 2^{d-2}=n 2^{d-2} .
$$

Hence by Lemma 2.3, $A_{\nu, n+1}$ has four pairwise non-isomorphic lifts over $W$ whose $F$-characters are as in (i),(ii), respectively $\left(\mathrm{i}^{\prime}\right),\left(\right.$ ii $\left.^{\prime}\right)$, if we replace $n$ by $n+1$. 
Proof of Theorem 1.2. Part (i) follows from Lemma 4.2, For part (ii), let $V$ be an arbitrary finitely generated endo-trivial $k D$-module. By Lemma 2.2 (ii), it is enough to determine $R(D, V)$ in case $V$ is indecomposable. This means by Remark 4.1 that $V$ is in the $\Omega$-orbit of $k$ or of $A_{\sigma, n}$ or of $A_{\tau, n}$ for some $n \geq 1$.

Let $\nu \in\{\sigma, \tau\}$ and let $n \geq 0$. It follows by Lemmas 5.3 and 5.4 that there is a surjective morphism

$$
\alpha: W[\mathbb{Z} / 2 \times \mathbb{Z} / 2] \rightarrow R\left(D, A_{\nu, n}\right)
$$

in $\mathcal{C}$ and that there are four distinct morphisms $R\left(D, A_{\nu, n}\right) \rightarrow W$ in $\mathcal{C}$. Hence $\operatorname{Spec}\left(R\left(D, A_{\nu, n}\right)\right)$ contains all four points of the generic fiber of $\operatorname{Spec}(W[\mathbb{Z} / 2 \times \mathbb{Z} / 2])$. Since the Zariski closure of these four points is all of $\operatorname{Spec}(W[\mathbb{Z} / 2 \times \mathbb{Z} / 2])$, this implies that $R\left(D, A_{\nu, n}\right)$ must be isomorphic to $W[\mathbb{Z} / 2 \times \mathbb{Z} / 2]$. By Lemma 2.2 , it follows that $R(D, V) \cong W[\mathbb{Z} / 2 \times \mathbb{Z} / 2]$ for every finitely generated endo-trivial $k D$-module $V$.

Let $U$ be a universal lift of $V$ over $R=R(D, V)$. Since $V$ is endo-trivial, the rank of $U$ as a free $R$-module is odd. This implies that as $R D$-modules

$$
U^{*} \otimes_{R} U \cong R \oplus L
$$

where $D$ acts trivially on $R$ and $L$ is some $R D$-module which is free over $R$. Since $U^{*} \otimes_{R} U$ is a lift of $V^{*} \otimes_{k} V$ over $R$ and $V$ is endo-trivial, this implies that $k \otimes_{R} L$ is isomorphic to a free $k D$-module. Hence $L$ is a free $R D$-module, which implies that $U$ is endo-trivial.

We now turn to nilpotent blocks and the proof of Corollary 1.3 .

Remark 5.5. Keeping the previous notation, let $G$ be a finite group and let $B$ be a nilpotent block of $k G$ with defect group $D$. By [9], this means that whenever $(Q, f)$ is a $B$-Brauer pair then the quotient $N_{G}(Q, f) / C_{G}(Q)$ is a 2-group. In other words, for all subgroups $Q$ of $D$ and for all block idempotents $f$ of $k C_{G}(Q)$ associated with $B$, the quotient of the stabilizer $N_{G}(Q, f)$ of $f$ in $N_{G}(Q)$ by the centralizer $C_{G}(Q)$ is a 2-group. In [25], Puig rephrased this definition using the theory of local pointed groups.

The main result of [25] implies that the nilpotent block $B$ is Morita equivalent to $k D$. In [26, Thm. 8.2], Puig showed that the converse is also true in a very strong way. Namely, if $B^{\prime}$ is another block such that there is a stable equivalence of Morita type between $B$ and $B^{\prime}$, then $B^{\prime}$ is also nilpotent. Hence Corollary 1.3 can be applied in particular if there is only known to be a stable equivalence of Morita type between $B$ and $k D$.

Proof of Corollary 1.3. Let $\hat{B}$ be the block of $W G$ corresponding to $B$. Then $\hat{B}$ is also nilpotent, and by [25, $\S 1.4], \hat{B}$ is Morita equivalent to $W D$. Suppose $V$ is a finitely generated $B$-module, and $V^{\prime}$ is the $k D$-module corresponding to $V$ under this Morita equivalence. Then $V$ has stable endomorphism ring $k$ if and only if $V^{\prime}$ has stable endomorphism ring $k$. Moreover, it follows for example from [4, Prop. 2.5] that $R(G, V) \cong R\left(D, V^{\prime}\right)$. By Theorem [1.2, this implies that $R(G, V) \cong W[\mathbb{Z} / 2 \times \mathbb{Z} / 2]$. 


\section{REFERENCES}

[1] J. L. Alperin, Local representation theory. Modular representations as an introduction to the local representation theory of finite groups. Cambridge Studies in Advanced Mathematics, vol. 11, Cambridge University Press, Cambridge, 1986.

[2] J. L. Alperin, Lifting endo-trivial modules. J. Group Theory 4 (2001), 1-2.

[3] M. Auslander and J. F. Carlson, Almost-split sequences and group rings. J. Algebra 103 (1986), 122-140.

[4] F. M. Bleher, Universal deformation rings and Klein four defect groups. Trans. Amer. Math. Soc. 354 (2002), 3893-3906.

[5] F. M. Bleher, Universal deformation rings and dihedral defect groups. In press, Trans. Amer. Math. Soc.

[6] F. M. Bleher and T. Chinburg, Universal deformation rings and cyclic blocks. Math. Ann. 318 (2000), 805-836.

[7] F. M. Bleher and T. Chinburg, Universal deformation rings need not be complete intersections. C. R. Math. Acad. Sci. Paris 342 (2006), 229-232.

[8] F. M. Bleher and T. Chinburg, Universal deformation rings need not be complete intersections, Math. Ann. 337 (2007), 739-767.

[9] M. Broué and L. Puig, A Frobenius theorem for blocks. Invent. Math. 56 (1980), 117-128.

[10] M. C. R. Butler and C. M. Ringel, Auslander-Reiten sequences with few middle terms and applications to string algebras. Comm. Algebra 15 (1987), 145-179.

[11] J. Byszewski, A universal deformation ring which is not a complete intersection ring. C. R. Math. Acad. Sci. Paris 343 (2006), 565-568.

[12] J. F. Carlson, A characterization of endotrivial modules over p-groups. Manuscripta Math. 97 (1998), 303-307.

[13] J. F. Carlson and J. Thévenaz, Torsion endo-trivial modules. Algebr. Represent. Theory 3 (2000), 303-335.

[14] J. F. Carlson and J. Thévenaz, The classification of endo-trivial modules. Invent. Math. 158 (2004), 389-411.

[15] J. F. Carlson and J. Thévenaz, The classification of torsion endo-trivial modules. Ann. of Math. (2) 162 (2005), 823-883.

[16] T. Chinburg, Can deformation rings of group representations not be local complete intersections? In: Problems from the Workshop on Automorphisms of Curves. Edited by Gunther Cornelissen and Frans Oort, with contributions by I. Bouw, T. Chinburg, G. Cornelissen, C. Gasbarri, D. Glass, C. Lehr, M. Matignon, F. Oort, R. Pries and S. Wewers. Rend. Sem. Mat. Univ. Padova 113 (2005), 129-177.

[17] E. Dade, Endo-permutation modules over p-groups. I; II. Ann. of Math. 107 (1978), 459-494; 108 (1978), 317-346.

[18] B. de Smit and H. W. Lenstra, Explicit construction of universal deformation rings. In: Modular Forms and Fermat's Last Theorem (Boston, MA, 1995), Springer-Verlag, Berlin-HeidelbergNew York, 1997, pp. 313-326.

[19] K. Erdmann, Blocks of Tame Representation Type and Related Algebras, Lecture Notes in Mathematics, vol. 1428, Springer-Verlag, Berlin-Heidelberg-New York, 1990.

[20] H. Krause, Maps between tree and band modules. J. Algebra 137 (1991), 186-194.

[21] M. Linckelmann, A derived equivalence for blocks with dihedral defect groups. J. Algebra 164 (1994), 244-255.

[22] M. Linckelmann, The source algebras of blocks with a Klein four defect group. J. Algebra 167 (1994), no. 3, 821-854.

[23] M. Linckelmann, On stable equivalences of Morita type. Derived equivalences for group rings, 221-232, Lecture Notes in Math., 1685, Springer, Berlin, 1998.

[24] B. Mazur, Deforming Galois representations. In: Galois groups over $\mathbb{Q}$ (Berkeley, CA, 1987), Springer-Verlag, Berlin-Heidelberg-New York, 1989, pp. 385-437. 
[25] L. Puig, Nilpotent blocks and their source algebras. Invent. Math. 93 (1988), 77-116.

[26] L. Puig, On the local structure of Morita and Rickard equivalences between Brauer blocks. Progress in Mathematics, 178. Birkhäuser Verlag, Basel, 1999.

[27] J. Thévenaz, $G$-algebras and modular representation theory. Oxford University Press, Oxford, 1995.

Department of Mathematics, University of Iowa, Iowa City, IA 52242-1419

E-mail address: fbleher@math.uiowa.edu 